\title{
Nanoindentation Induced Elastic-plastic Deformation of GaN Nanomembrane on a Sapphire Substrate
}

\author{
Maha Khayyat ${ }^{1, ~ *, ~ E r i c ~ B o u r h i s ~}{ }^{2}$, Rami Elafandy ${ }^{3}$, Tienkhee $\mathrm{Ng}^{3}$, Boon Ooi $^{3}$ \\ ${ }^{1}$ Nanotechnology and Semiconductors Center, Materials Science Research Institute, King Abdullaziz City for Science and Technology \\ (KACST), Riyadh, Saudi Arabia \\ ${ }^{2}$ Department of Physics and Mechanics of Materials, Institute P', University of Poitiers, Poitiers, France \\ ${ }^{3}$ Computer, Electrical \& Mathematical Sciences \& Engineering Division, King Abdullah University for Science and Technology (KAUST), \\ Thuwal, Saudi Arabia
}

Email address:

mkhayyat@kacst.edu.sa (M. Khayyat)

${ }^{*}$ Corresponding author

\section{To cite this article:}

Maha Khayyat, Eric Bourhis, Rami Elafandy, Tienkhee Ng, Boon Ooi. Nanoindentation Induced Elastic-plastic Deformation of GaN Nanomembrane on a Sapphire Substrate. American Journal of Nanosciences. Vol. 7, No. 1, 2021, pp. 15-22. doi: 10.11648/j.ajn.20210701.13

Received: January 4, 2021; Accepted: January 19, 2021; Published: January 28, 2021

\begin{abstract}
One of the main challenges of the production of a blue laser is the preparation of defect-free GaN layers. It is of high tehnological interest to characterize GaN nanomembrane mechanically for further advanced applications. The current study addresses the impact of applied stresses on $\mathrm{GaN}$ nanomembranes, which are placed on sapphire the substrates, using nanoindentation as a nondestructive test. The mechanical response of the 20 , and $100 \mathrm{~nm}$ thin GaN nanomembrane were studied at different normal applied loads ranging from $1 \mathrm{mN}$ down to $0.1 \mathrm{mN}$ using the Berkovich nanoindentation technique. There were plastic deformation regions at the nanoindented $\mathrm{GaN}$ nanomembranes monitored by the load-displacement $(p-h)$ curves. The depth of the deformed regions increased with increasing the applied loads on the diamond indenter. Beside the insitu depth estimation of the residual nanoindentation using the instrumented nanoindentation machine, Atomic Force Microscopy (AFM) has been deployed as an ex-situ measurements of indentations depth. Scanning Electron Microscopy (SEM) provided us with surface images of the indented membranes. Indentation of the $100 \mathrm{~nm}$ thick GaN nanomembrane, where the effect of the substrate is reduced, showed discontinuity in the $p$ - $h$ curves. These discontinuity or pop-in events were attributed to a possible sudden initiation and propagation of threading dislocations in the GaN nanomembrane which was free of threading dislocation upon fabrication. It was suggested to employ $\mu$-Raman spectroscopy methods to investigate the possible structural phase transformation of thicker GaN nanomembranes and to measure the compressive or tensile stresses within the center of the indented zones. Where the observed sudden load-displacements discontinuity or depth excursions during indentation of GaN nanomembranes can be attributed.
\end{abstract}

Keywords: Nanoindentation, GaN, Nanomembrane, Sapphire, AFM

\section{Introduction}

$\mathrm{GaN}$ is one of the most important optoelectronic semiconductors for its applications as white light source, blue diode leading to UV lithography.3 High dislocation density and related issues in $\mathrm{GaN}$ are one among few of the major hindrances for the application as blue laser. 4 Thus, removal of stress is one of the prime objectives for opto-electronic device applications in $\mathrm{GaN}$ [1-3]. $\mathrm{GaN}$ is a wide band-gap III-V compound semiconductor. It usually has a wurtizitic structure
$(\alpha-\mathrm{GaN})$ with a direct band gap $3.4 \mathrm{eV}$ at room temperature, which offers the potential for fabricating a variety of near ultraviolet (UV) optoelectronic devices, such as blue light emitting diodes [2,3]. The successful fabrication of devices based on epitaxial GaN thin films requires better understanding of their mechanical characteristics, since the contact loading during processing or packaging can significantly degrade the performance of these devices [4]. Moreover, such devices will experience large thermal strains in addition to the growth strains leading to cracking thus posing problems for the development of $\mathrm{GaN}$ based devices. Therefore, there is a growing demand of 
investigating the mechanical characteristics of materials, in particular in the nanoscale regime, for device applications $[5,6]$. More particularly, however, contact loading is a type of mechanical impact that semiconductor materials often inevitably experience during processing. This kind of loading may cause structural phase transformations and consequently change the band gap of the material [4]. Hence, the study of mechanical behaviour of $\mathrm{GaN}$ nanomembranes is the key research topic for their successful device application.

The indentation test represents a simple and quite unique approach for studying of metastable high-pressure phases of semiconductors, in addition to the mechanical properties of solid materials [4]. A combination of biaxial and hydrostatic stresses, as in the case of indentation, originating form dislocation related extended defects and point defects, respectively, are reported generally in epi-GaN [5]. Where Daimond Anvil Cells (DAC), shown only the effect of hydrostatic pressures. However, hydrostatic stress alone is estimated inside the indented region of epi-GaN without implication of any structural changes.

Lim et. al [7] explained indentation-induced plastic deformation under a pointed indenter for an isotropic solid. A theoretical study was carried out by another research group at University of Cambridge, Needs et al, 2003 on high pressures phases of group-IV, III-V and II-VI compounds, including $\mathrm{GaN}$ [8]. The plastic deformation of the III-V semiconductors under concentrated load was reviewed by Le Bourhis and Patriarche [9]. Indentation experiments involves pressing a geometrically well-defined piece of a very hard material, such as diamond, into another solid under a known load, creating very high stresses, both hydrostatic and shear, in the material indented [4]. For the purpose of studying the mechanical properties of materials and other related properties of thin films nanoindentation technique should be employed. Instrumental nanoindentation allows monitoring the penetration of a diamond tip as a function of the applied load. Moreover, it offers us a method to monitor the threshold of plastic deformation by applying ultra-low loads, so we can avoid fracture as explained by Le Bourhis and Patriarche [10]. Using the instrumented nanoindenter one can deduce information about the elastic-plastic response of the thin film as well as their adhesion to the substrate $[9,10]$. If a material underneath the indenter transforms into a new phase when certain stress conditions are met, changes in the slope of the loading/unloading curve or discontinuities in the loading/unloading curves are expected [11]. Previous work of mechanical properties of $\mathrm{GaN}$ thin films were carried out on relatively thick films, Jian [12] nanoindented $2 \mu \mathrm{m}$ thick films grown on sapphire by MOCVD method, using TEM and CL techniques. Also, Lin et al [13] examined a $2 \mu \mathrm{m}$ thick GaN thin film using nanoindentation and AFM tehniques. Navamathavan et. al [14] carried out nanoindentation on 1-4 $\mu \mathrm{m}$ thick GaN thin films on sapphire. Geetha et al [15] carried nanoindentation experiments on $2 \mu \mathrm{m}$ films of $\mathrm{GaN}$ and examined the residual microindentation using AFM. Bradby et al [16] studied the mechanical deformation of wurtzite $\mathrm{GaN}$ epilayers grown on sapphire substrates using spherical nanoindntation and cathodoluminescence (CL). In 2007 Khayyat et. al [17] studied the effect of temperature on structural phase tramsformation of $\mathrm{Si}$, and recently in 2020 detailed simulation [18] and experimental [19] studies were carried out on the impact of temperature and growth parameters on GaN crystals during indentation which showed that strain increased with increasing temperature and growth conditions of the crystalline GaN films.

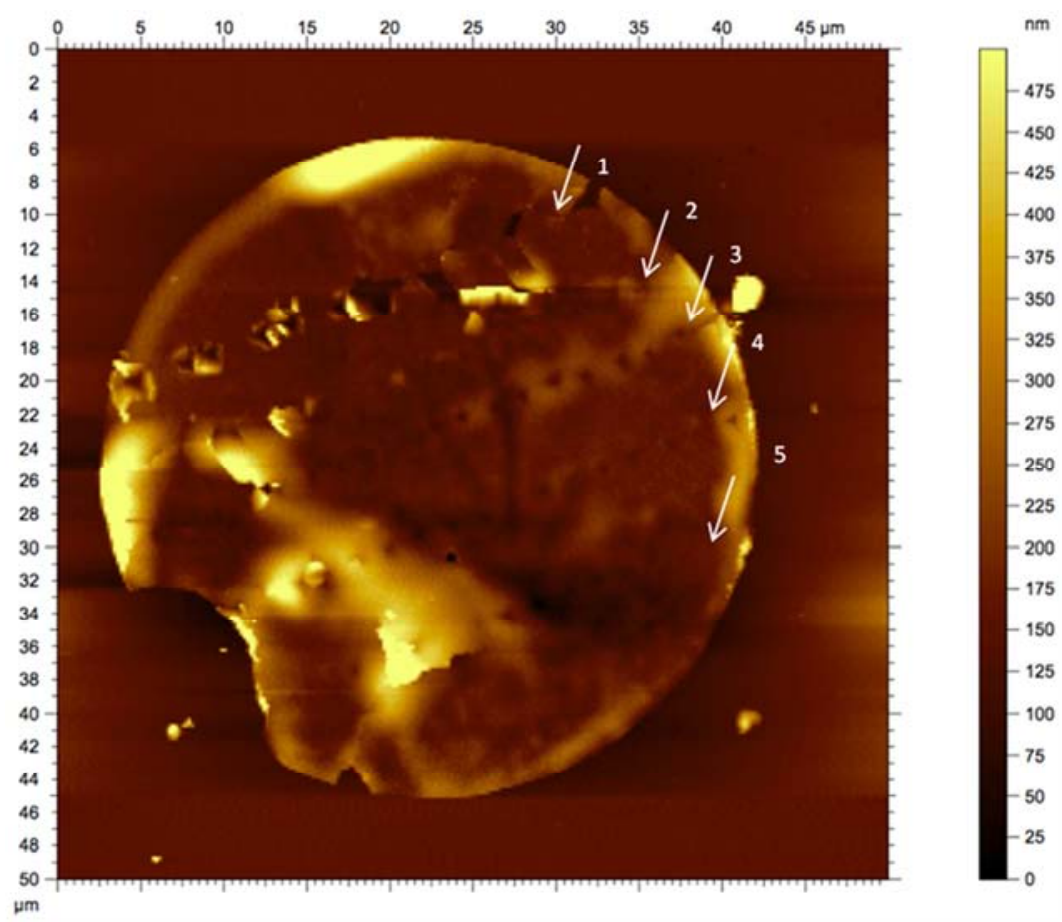

Figure 1. AFM image shows the $20 \mathrm{~nm}$ thick GaN nanomembrane on a sapphire substrate at various Berckovish indenter loads: first row marked with the arrow 1 shows indentation at $20 \mathrm{mN}$, arrow 2 at $1 \mathrm{mN}$, arrow 3 at $0.5 \mathrm{mN}$, arrow 4 at $0.2 \mathrm{mN}$, arrow 5 at $0.1 \mathrm{mN}$. 


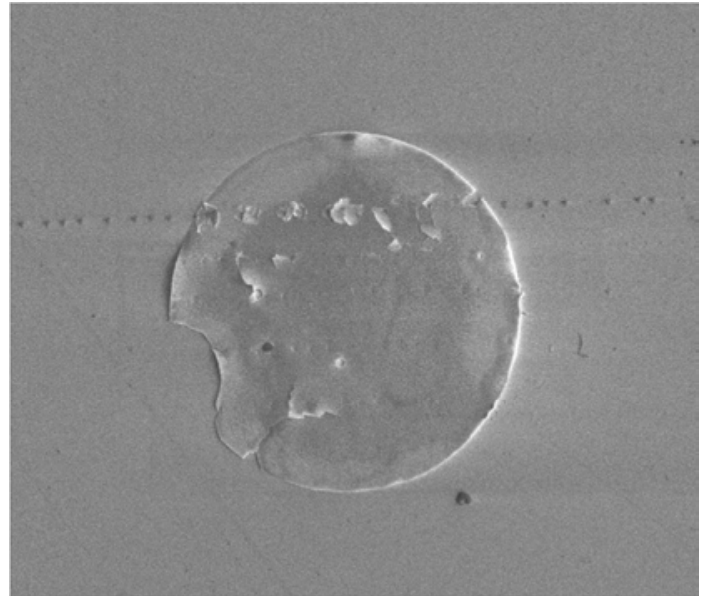

Figure 2. SEM micrograph of $20 \mathrm{~nm}$ thick GaN nanomembrane after undertaken nanoindentation, as shown in Figure 1 using AFM.

\section{Experimental}

The process of fabrication the GaN nanomembranes was explained by Rami Elafandy et. al [20]. GaN nanomembranes of $20 \mathrm{~nm}$ and $100 \mathrm{~nm}$ thick and $50 \mu \mathrm{m}$ diameter were prepared by chemical exfoliation of completely threading dislocation free single crystalline GaN nanomembranes, using UV-assisted electroless chemical etching. The GaN wafers were grown using hypride vapor phase epitaxy (HVPE) technique. The details of growth procedures in preparing these $\mathrm{GaN}$ can be found elsewhere [20-22]. Then exfoliated GaN nanomembranes were transferred mechanically by lifting them with a $40 \mathrm{~nm}$ thick tungsten probe then place them on a sapphire substrate [20].

Nanoindentations were carried out using the Nano-Hardness Tester CSEM Instrument, fitted with Berkovich diamond indenter (serial number; BC-33-SCB2) [24]. The $20 \mathrm{~nm}$ thick $\mathrm{GaN}$ nanomembrane was indented with a Berkovich indenter with peak loads of 20,1, 0.5, 0.2, $0.1 \mathrm{mN}$ (see Figures $1 \& 2$ ) at room temperature with a dwell time of $30 \mathrm{~s}$, similarly the pausing and unloading time, where the rate varies according to the maximum normal applied loads. Instrumented nanoindentation allows for monitoring the penetration of a tip into a specimen as a function of load. The Young's modulous and hardness of these samples were determined from the loaddepth curves using the method of Oliver and Pharr [25]. Similarly, The $100 \mathrm{~nm}$ thick GaN nanomembrane was indented at $0.5 \mathrm{mN}$ and $0.2 \mathrm{mN}$. The samples to be examined were mounted on a glass cylinder. The mounted sample was placed on a flat base of the $x-y-z$ translation stage of the machine to undertake nanoindentation at various normal indenter loads.

\section{Results and Discussion}

First, nanoindentation experiments were carried out on sapphire substrate to examine the elastic zone so we can examine the elastic-plastic response of GaN NM without an interference of the substrate. A representative loadingunloading curve of sapphire is shown in Figure 3 (a), where the maximum normal applied load was $20 \mathrm{mN}$, applied at the $20 \mathrm{~nm}$ thick GaN nanomembrane. The average load is $13 \pm 1.16 \mathrm{mN}$ where pop-in occurs. The average hardness is $42.10 \mathrm{GPa}$, the hardness values have been measured using the projected contact areas obtained from Oliver \& Pharr method [25]. This value of hardness is in general agreement with the previous reported measurements, Mao et al. [26] showed that the hardness of $C$ plane of sapphire is $46.7 \pm 15$ $\mathrm{GPa}$. On the other hand, there is a discrepancy in the published value of sapphire hardness, as Lim et al. [7] reported the value of the sapphire hardness to be $26 \mathrm{GPa}$. A curve obtained from indenting the surface of the sapphire substrate with $20 \mathrm{mN}$ normal load. It shows an elastic loading up to $13 \pm 1.16 \mathrm{mN}$ where a discontinuity or a pop-in is observed on loading, and the unloading remains elastic.

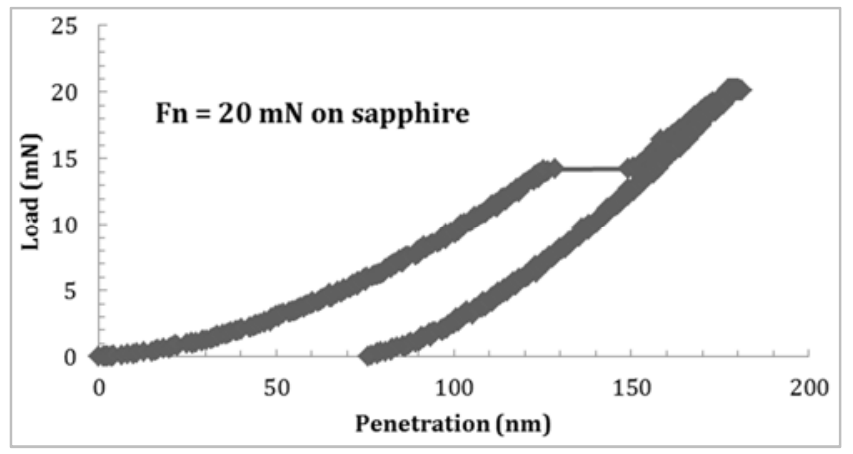

(a)

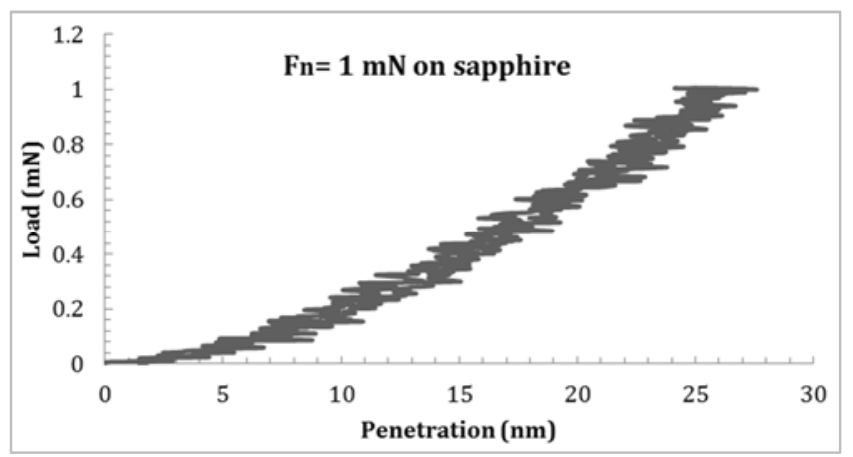

(b)

Figure 3. (a) a representative curve of indenter load (y) in $m N$ vs. penetration depth $(x)$ in $\mathrm{nm}$ of a Bercovich indenter into sapphire substrate showing the pop-in or excursion event for an indenter load of $13 \pm 1.16 \mathrm{mN}$. (b) Loading and unloading curves of a Berkovich indenter in the sapphire at room temperature under $1 \mathrm{mN}$.

At this relatively high load of $20 \mathrm{mN}$, the $\mathrm{GaN}$ nanomembrane shuttered into flakes upon nanoindentation, and the indenter tip penetrates to the substrate. Based on that, we excluded the measurements of $20 \mathrm{mN}$ nanoindentation of the $20 \mathrm{~nm}$ thick GaN nanomembrane. Nanoindenting the 20 $\mathrm{nm}$ thick GaN nanomembrane at $1 \mathrm{mN}$ shows that there is not any plastic deformation, as it confirmed by the loadingunloading curve (see Figure 3 (b)). The slope of the loading curve of sapphire, at $\mathrm{Fn}=1 \mathrm{mN}$, almost coincides with the slope of the unloading curve. 
Figures 5 and 6 show AFM images and profiles of $1 \mathrm{mN}$ and $0.1 \mathrm{mN}$ nanoindentations of $20 \mathrm{~nm}$ thick GaN nanomembrane. The image of $1 \mathrm{mN}$ nanoindentation shows perfect impressions without any trace of radial crack is observed, similarly, the $0.1 \mathrm{mN}$ nanoindentation shows no cracks, as one would expect at this ultra-low indenter loads. The AFM profiles of $1 \mathrm{mN}$ and $0.1 \mathrm{mN}$ (see Figures 5\&6) show material pile-up near the indented zones, revealing the elastic-plastic behaviour of the $20 \mathrm{~nm}$ thick GaN nanomembrane [15].

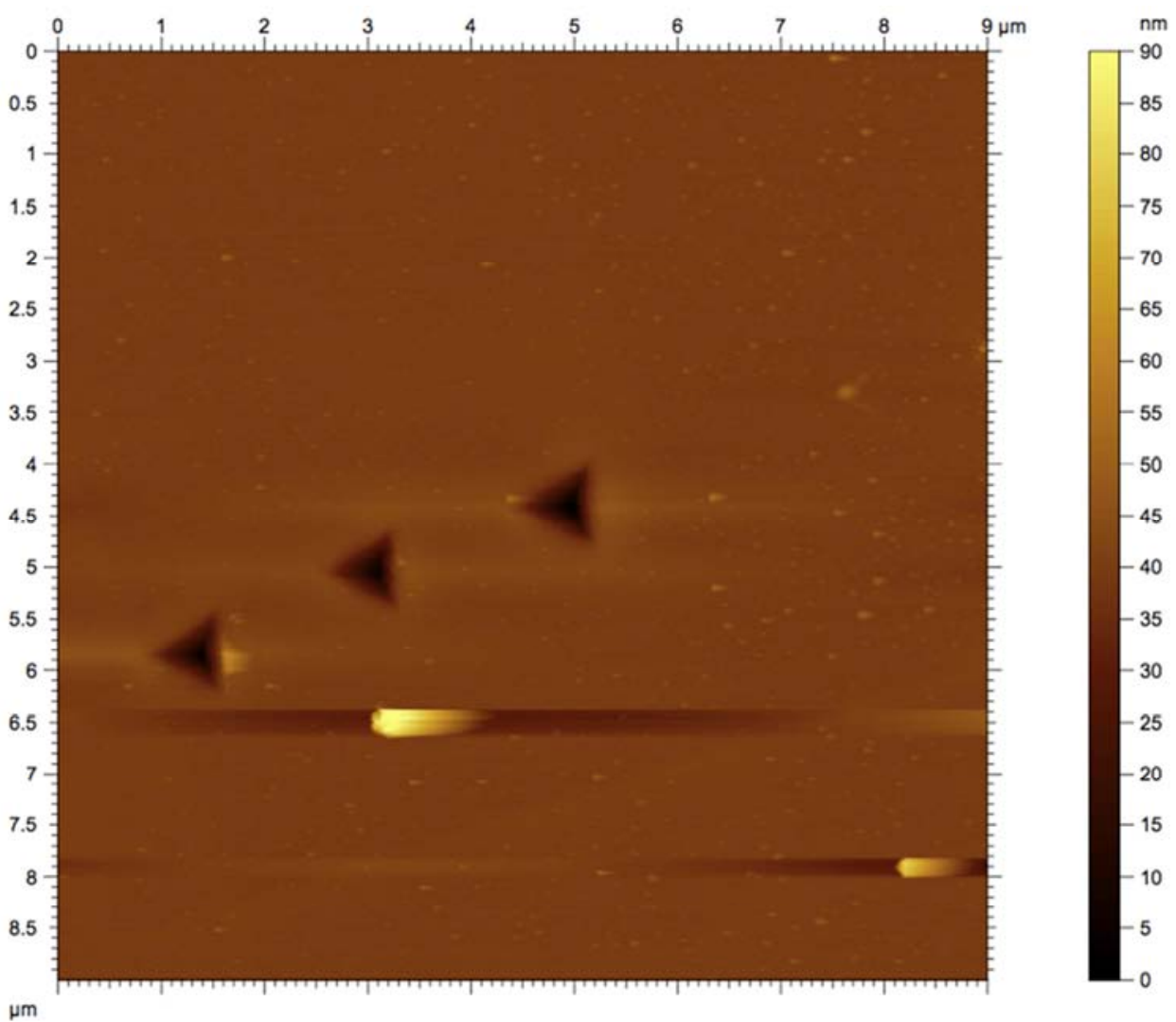

(a)

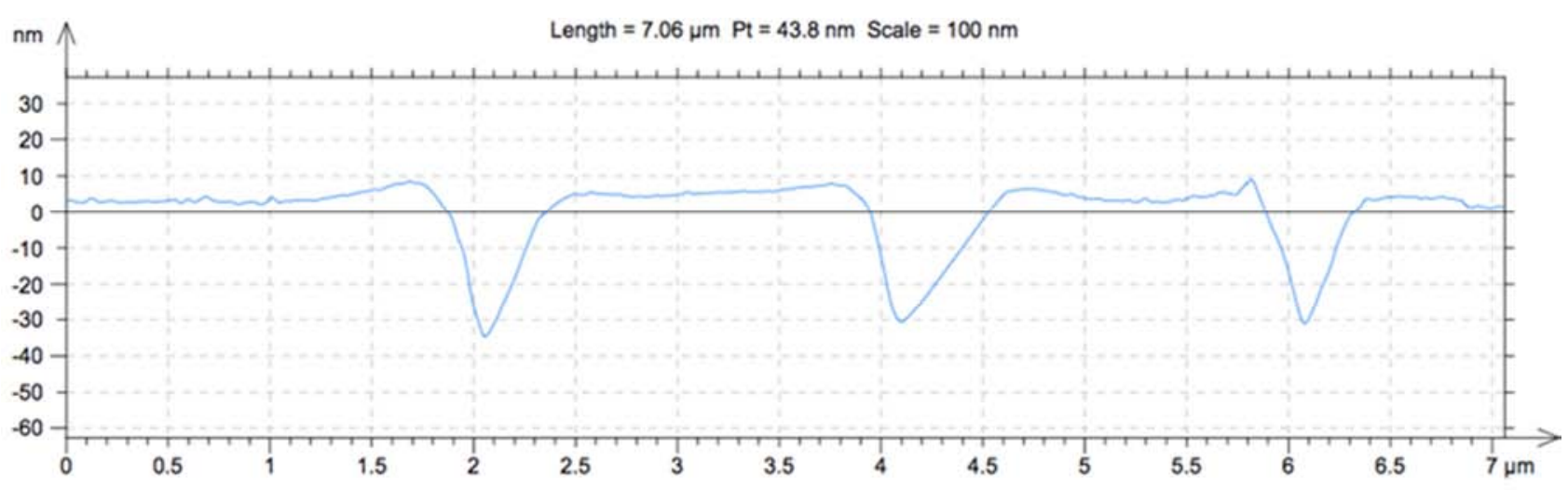

(b)

Figure 5. (a) $1 \mathrm{mN}$ nanoindentations on $20 \mathrm{~nm}$ thick GaN nanomembrane. It shows perfect impressions without any traces of radial cracks are observed. (b) nanoindentations profile.

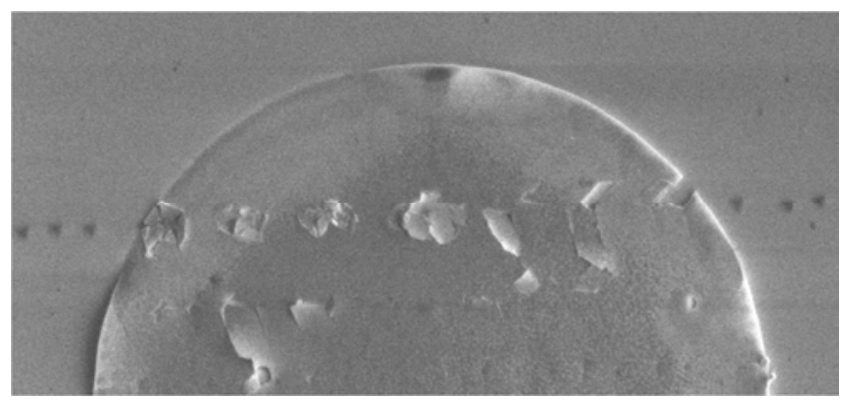

Figure 4. The $20 \mathrm{~nm}$ thick GaN nanomebrane after indentening at $10 \mathrm{mN}$ indenter load, see the flakes of the GaN nanombrane at the nanoindented zones. 


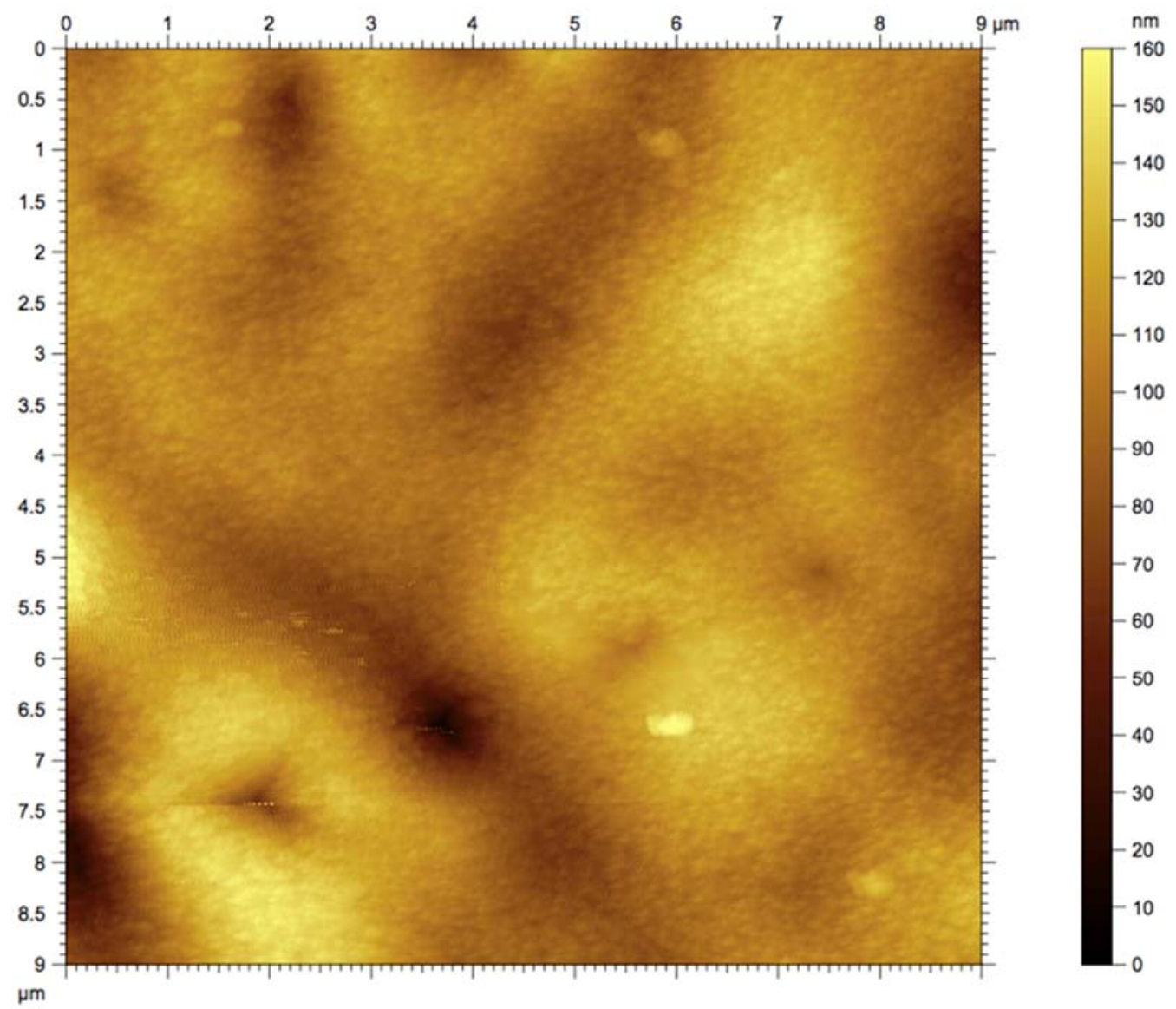

(a)

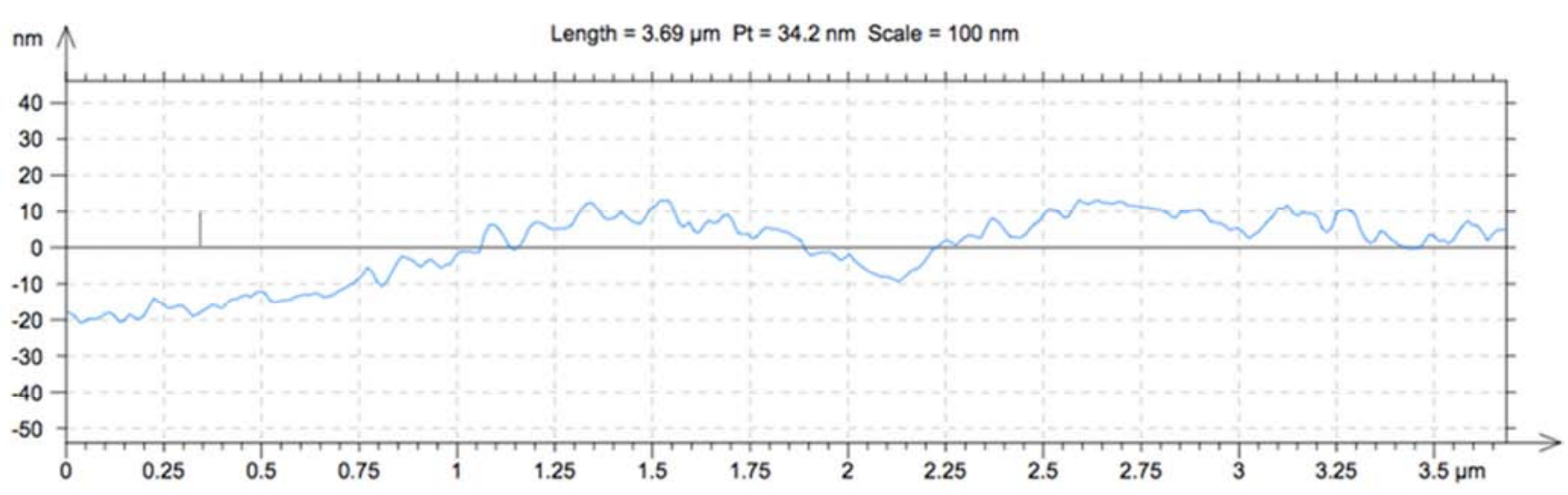

(b)

Figure 6. (a) AFM image of $0.1 \mathrm{mN}$ indenter load nanoindentation of $100 \mathrm{~nm}$ thick GaN nanomembrane. (b) nanoindentation profile.

Figure 7 (a:c) shows the load-penetration curves of nanoindentation on $20 \mathrm{~nm}$ thick $\mathrm{GaN}$ nanomembrane at ultra- low loads. As it is shown in the load-penetration curves, as the indenter load increases, $0.1 \mathrm{mN}, 0.2, \mathrm{mN}, 0.5$ $\mathrm{mN}, 0.1 \mathrm{mN}$, the penetration depth increases. It is important to note that all these values remain below the membrane thickness indicating that the interface between the membrane and the substrate is not reached by the indenter tip extremity.

Nanoindenting the $100 \mathrm{~nm}$ thick GaN nanomembrane show elastic-plastic deformation as shown in Figure 8.
Typical nanoindentation $\mathrm{p}-\mathrm{h}$ curves of $\mathrm{GaN}$ nanomembrane subjected to the maximum indentation loads of $0.5 \mathrm{mN}$ and $0.2 \mathrm{mN}$. The curves exhibit irregularities during loading characterized by pop-in. The frequency of such events was estimated to be about $60 \%$. Such discontinuities correspond to a sudden deformation of the sample and generally attributed to the onset of plasticity.

The curves (Figure 8 a $\&$ b) exhibit a single discontinuity at an indentation depth of $27 \mathrm{~nm}$ corresponding to the applied load of $0.5 \mathrm{mN}$, and $30 \mathrm{~nm}$ at $0.2 \mathrm{mN}$. No other pop-in event was observed when indentation load was further increased. 
Lin et al. [13] observed pop-in events took place at $16 \mathrm{~nm}$ at applied load $0.16 \mathrm{mN}$, and attributed the discontinuity or pop-in to dislocation associated phenophmenon. Navamathavan [6] observed a single pop-in event at $0.4 \mathrm{mN}$ indenter load, penetration depth $20 \mathrm{~nm}$ of $\mathrm{GaN}$ thin films (1$4 \mu \mathrm{m}$ thick). They attributed the pop-in to the lattice mismatch of the GaN epitaxial film with respect to the substate or/and sudden propagation of threading dislocation [4]. It was observed by Bradby [16] there are discontinuity observed during loading when the maximum load is above

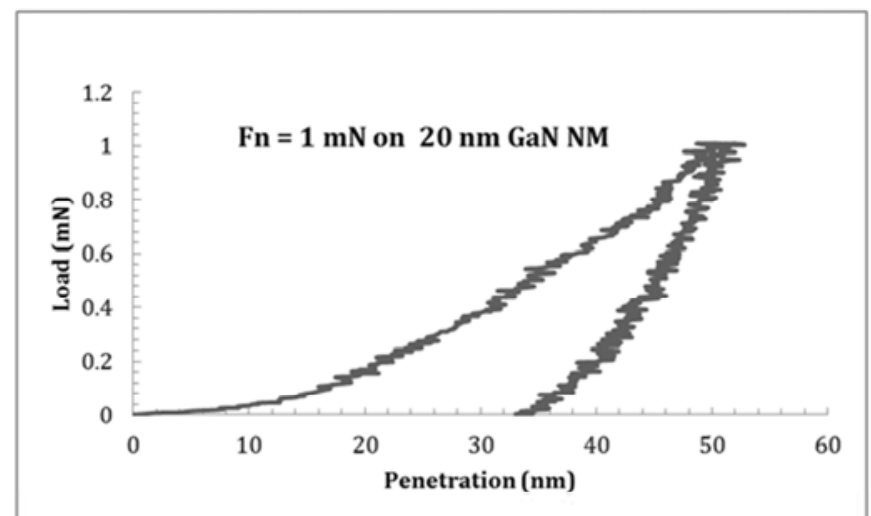

(a)

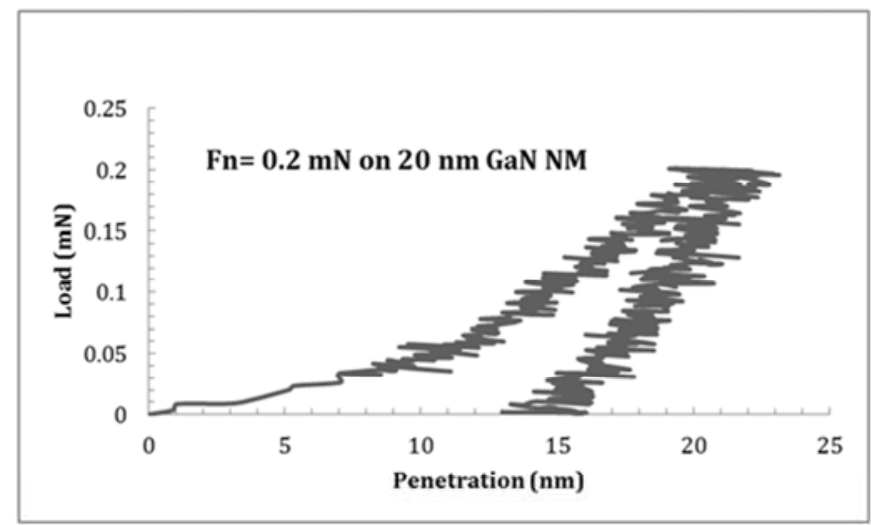

(c) the elastic-plastic threshold. This behavior was correlated by Bradby et al. with multible slip bands revealed by XTEM [16]. The physical mechanism responsible for the 'pop-in' event may be due to the interaction behavior of the indenter tip with the pre-existing threading dislocation present in the films during mechanical deformation [27]. It was observed that the 'pop-in' depth is corelated with the lattice mismatch of the epitaxial thin film with the substrate, the higher the lattice mismatch the shallower the critical 'pop-in' depth [27, 28].

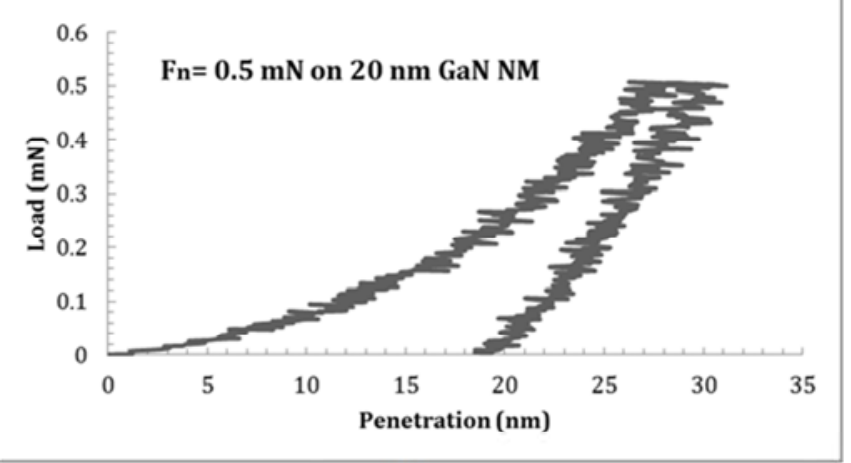

(b)

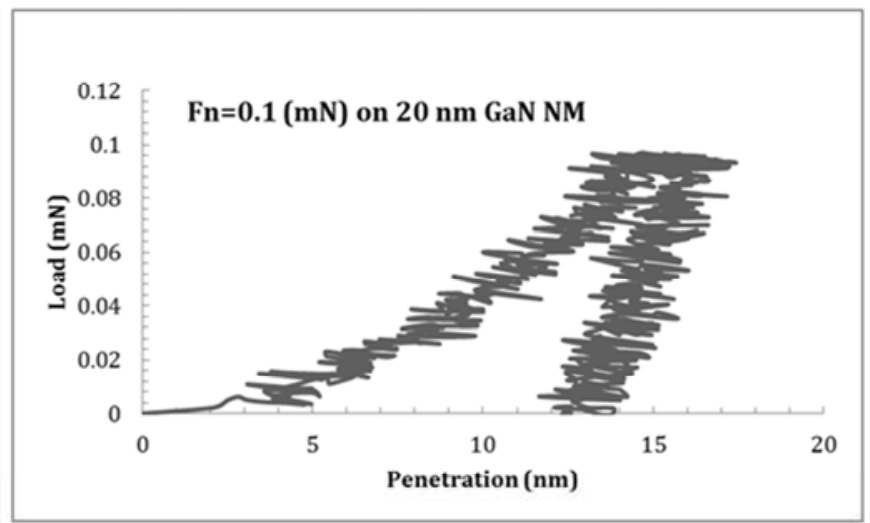

(d)

Figure 7. (a) Loading and unloading curves of a Berkovich indenter on $20 \mathrm{~nm}$ thick $G a N$ nanomebrane at $1 \mathrm{mN}$. (b) at $0.5 \mathrm{mN}$ normal indenter load. (c) at 0.2 $\mathrm{mN}$. (d) at $0.1 \mathrm{mN}$.

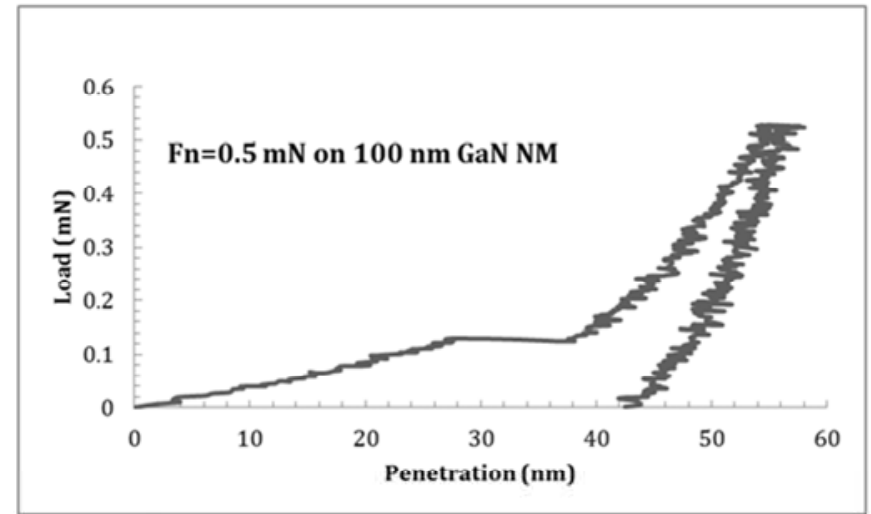

(a)

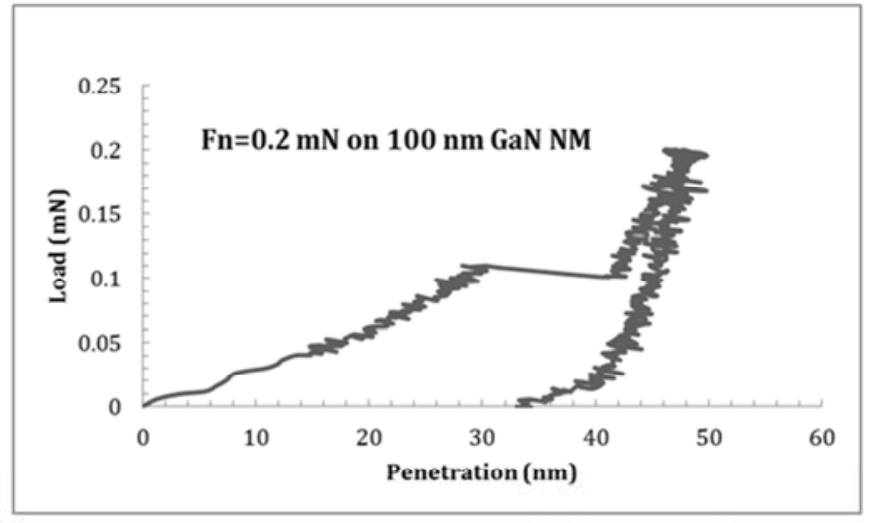

(b)

Figure 8. (a) load vs. penetration curve of a Berkovich indenter into $100 \mathrm{~nm}$ GaN nanomembrane for a load of $0.5 \mathrm{mN}$. (b) at $0.2 \mathrm{mN}$. 
Table 1. Shows the Vickers hardness value of the $20 \mathrm{~nm}$ and $100 \mathrm{~nm}$ thick GaN nanomembrane at various applied loads. The fluctuations in the value of the hardness of GaN crystals can be explained by the growth conditions induce a change in the nitrogen vacancy concentration and consequently the hardness value $[18,28]$.

\begin{tabular}{lll}
\hline Indenter load $(\mathbf{m N})$ & Hv $(\mathbf{G P a}) \mathbf{2 0} \mathbf{~ n m}$ thick GaN NM. & Hv (GPa) 100 nm thick Ga NM. \\
\hline 1 & 5.46 & N/A \\
0.5 & 8.87 & 5.07 \\
0.2 & 6.12 & 2.54 \\
0.1 & 3.98 & N/A \\
\hline
\end{tabular}

* not available

The pop-in event was attributed to the lattice mismatch of the epitaxial films with respect to the substrate [23, 27]. Other possible mechanism that would explain the observed behaviour is the formation and propagation of dislocations in $\mathrm{GaN}$ thin films [28]. GaN grown on sapphire substrates suffer from high density of threading dislocation [29, 30]. However, the GaN nanomembranes examined in the current study are threading dislocation (TD) free as it confirmed by TEM measurements [20]. Based on that, the pop-in events cannot be attributed to the extension of the TD pre-existed in the film. It might be a sign of an initiation of TD within the nanomembranes. On the other hand, this observation of the discontinuity event was observed in $\mathrm{Si}$, and it has been linked to phase transformations [11]. However, to investigate the possible structural phase transition within the center of the indented zones, one should undertake a relatively high load at thicker membranes to apply the laser beam of a controlled diameter and measure the Raman scattering spectra [31].

Nanoindentation was employed to study the mechanical properties of thin films [32], such as the GaN naomembranes under consideration.

\section{Conclusion}

The elastic-plastic behaviour of materials associated with various microstructures, micro/nano-mechanic techniques such as nanoindentation have been well established and widely used. Based on this, the mechanical deformation behaviour of $\mathrm{GaN}$ nanomembranes placed on sapphire substrate was studied using nanoindentation. At high loads there are cracking and fragmentation was observed at $\mathrm{GaN}$ nanomembranes. Nanoindentations at ultra-ow loads, ranging from $1 \mathrm{mN}$ to $0.1 \mathrm{mN}$ show perfect impressions without any traces of radial cracks. Load-displacement curves of both the $20 \mathrm{~nm}$ and $100 \mathrm{~nm}$ thick GaN nanomembranes show elasticplastic deformation as it was exhibited by the change of the slope of the loading-unloading curves. Moreover, the $100 \mathrm{~nm}$ thick GaN nanomembrane show a single pop-in event at the loading curve on 0.5 and $0.2 \mathrm{mN}$ applied loads. The excrusion or the pop-in was attributed to the possible initiation of threading dislocation (TD) in the nanomembrane which was TD free upon fabrication. The Vickers hardness value of the $20 \mathrm{~nm}$ thick GaN nanomembrane is higher than that of the $100 \mathrm{~nm}$ thick nanomembrane. Clearly, the $20 \mathrm{~nm}$ thick GaN hardness measurements was affected by the hard substrate. To investigate the possible phase transition in the $\mathrm{GaN}$ nanomembrane, nanoindentation should be undertaken at slightly thicker membrane to be monitored by the micro Raman spectroscopy.

\section{References}

[1] R. Garcia, B. Ren, A. C. Thomas, and F. A. Ponce, Measurement of the solubility of ammonia and nitrogen in gallium at atmospheric pressure, J. of Alloys and Compounds, 2009, 467, 611-613.

[2] J. N. Dai, X. H. Wu, C. H. Yu, Q. Zhang, Y. Q. Sun, Y. K. Xiong, X. Y. Han, L. Z. Tong, Q. H. He, F. A. Ponce, and C. Q. Chen, Comparative study on MOCVD growth of a-plane GaN film on r-plane sapphire substrates using $\mathrm{GaN}, \mathrm{AlGaN}$, and AlN buffer layers, J. of Electronic Materials, 2009, 38, 19381943.

[3] T. Onuma, A. Uedono, H. Asamizu, H. Sato, J. F. Kaeding, M. Iza, S. P. DenBaars, S. Nakamura, and S. F. Chichibu, Photoluminescence and positron annihilation studies on $\mathrm{Mg}$ doped nitrogen-polarity semipolar (1011) GaN heteroepitaxial layers grown by metalorganic vapor phase epitaxy, APL, 96, 091913 (2010).

[4] Maha Mohammed Omar Khayyat, Studies of Indentationinduced phase transformations in semiconductors, $\mathrm{PhD}$ dissertation 2004, University of Cambridge, Cavendish laboratory.

[5] W. C. Oliver, G.M. Pharr, Journal of Materials Research 7 (1992) 1564-1583.

[6] R. Navamathavan, Seong-Ju Park, Jun-Hee Hahn, Chi Kyu Choi, A nanoindentation analysis of the influence of lattice mismatch on some wide band gap semiconductor films, Physica B 403 (2008) 675-678.

[7] Y. Y. Lim, M. M. Chaudhri, and Y. Enomotto, Accurate determination of the mechanical properties of thin aluminum film deposited on sapphire flats using nanoindentation, J. Mater. Rese., Vol. 14, No. 6, 1990, 2314.

[8] A. Mujica, Angel Rubio, A. Munoz and R. J. Needs, High pressure phases of group-IV, III-V, and II-VI compounds, Review of Modern Physics, Vol 75, 2003, p.863-911.

[9] E. Le Bourhis, G. Patriarche, Plastic deformation of III-V semiconductors under concentrated load, Progress in Crystal Growth and Characterisation of Materias (2003) 1-43.

[10] E. Le Bourhis and G Patriarche, Nanoindentation response of a thin InP membrane, J. Phys. D: Appl. Phys. 41 (2008) (5pp).

[11] Yury G. Gogotsi, Vladislav Domnich, Sergey N. Dub, Andreas Kailer and Klaus G. Nickel, Cyclic nanoindentation and Raman microscopy study of phase transformations in semiconductors, J. Mater. Res. Vol. 15, No. 4, 2000. 
[12] Sheng-Rui Jian, Berkovich indentation-induced deformation behaviors of $\mathrm{GaN}$ thin films observed using cathodoluminescence and cross sectional transmission electron microscopy, Applied Surface Science 254 (2008) 6749-6753.

[13] Meng-Hung Lin, Hua-Chiang Wen, Chih-Yung Huang, YeauRen Jeng, Wei-Hung Yau, Wen-Fa Wu, Chang-Pin Chou, Nanoindentation characterization of GaN epilayers on A-plane sapphire substrate, Appl. Surf. Scie. 256 (2010) 3464-3467.

[14] R. Navamathavan, Seong-Ju Park, Jun-Hee Hahn, and Chi Kyu Choi, Physica B 403 (2008), p675-678.

[15] D. Geetha, D. Arivuoli, G. Mangamma, AFM studies of microindented $\mathrm{GaN}$ and InGaN, Materials Letters 63 (2009) 515-518.

[16] J. E. Bradby, S. O. Kucheyev, J. S. Williams, J. Wong-Leung, M. V. Swain, P. Munroe, G. Li and M. R. Phillips, Indentationinduced damage in GaN epilayers, Appl. Phys. Lett., 80, 383 (2002).

[17] M. M. O. Khayyat, D. G. Hasko, and M. M. Chaudhri, Effect of sample temperature on indentation-induced phase transitions in crystalline silicon J. Appl. Phys. 101, 083515 (2007).

[18] Jian Guo, Jinging Chen, Yongqiang Wang, Temperature effect on mechanical response of c-plane monocrystalline gallium nitride in nanoindentation: A molecular dynamics study, Ceramics Intl 46 (2020) 12686-12694.

[19] S. A. Garcia Hernandez, V. D. Compean Garcia, E. Lopez Luna, M. A. Vidal, Elastic modulus and hardness of cubic $\mathrm{GaN}$ grown by molecular beam epitaxy obtained by nanoindentation, This Solid Films 699 (2020) 137915.

[20] Rami T. El-Afandy, Mohammed A. Majid, Tien Khee Ng, Lan Zhao, Dongkyu Cha, and Boon S. Ooi, Exfoliation of Threading Dislocation-Free, Single-Crystalline, Ultrathin Gallium Nitride Nanomembranes, Adv. Funct. Mater. 2013, 17.

[21] Hajima Fuujikura, Yuichi Oshima, Takeshi Megro, Toshiya Saito, Journal of Crystal Growth 350 (2012), p 38-43.
[22] Tae Su Oh, et al., Journal of Alloys and Compounds 509 (2011), p2952-2956.

[23] S. Ohira, and N. Arai, Phys. Stat. Sol. (c) 2008, 5, 3116.

[24] Indentation Software, CSEM instrument, version 1.4, User's Guide. http://www.csem.ch/instrum/.

[25] W. C. Oliver, and G. M. Pharr, An improved technique for determining hardness and elastic modulous using load and displacement sensing indentation experiments, J. Mat. Res. 7(6), 1992, 1564-1583.

[26] W. G. Mao, Y. G. Shen, C. Lu, Nanoscale elastic-plastic deformation and stress distribution on the $\mathrm{C}$ plane of sapphire single crystal during nanoindentation, J. Euro. Ceram. Soci. 31 (2011) 1865-1871.

[27] F. G. Torres, E. Le Bourhis, O.P. Troncoso, and J. Liamoza, Structural-Prperty Relationships in Arapaima Gigas Scales Revealed by Nanoindentation Tests, Polymers \& Polymer Composites, Vol. 22, No. 4, 2014.

[28] Sheng-Rui Jian, Berkovich indentation-induced deformation behaviours of $\mathrm{GaN}$ thin films observed using cathodoluminescenece and cross-sectional transmission electron microscopy, Appl. Surf. Scie. 254 (2008), p. 6749.

[29] M. Munawar Chaudhri, Dislocations and Indentations 12, Edited by F. R. N. Nabarro and J. P. Hirth (Elsevier Science, 2004).

[30] Glass Mechanics and Technology, Eric Le Bourhis, WileyYCH publisher, 2008, Appendix 8, p. 291.

[31] G. Patriarche, E. Le Bourhis, M. M. O. Khayyat, and M. M. Chaudhri, Indentation-induced crystallization and phase transformation of amorphous germanium, JAP, 96, 3, 2004, p. 1464-1468.

[32] A. S. Grashchenko, S. A. Kukushin, A. V. Osipov, A. V. Redkov, Nanoindentation of $\mathrm{GaN} / \mathrm{SiC}$ thin films on silicon substrate, J. of Physics and Chemistry of Solids 102 (2017), 151-156. 